\title{
Investigação de Etiologia Genética nas Ataxias Neurodegenerativas: Recomendações do Grupo de Neurogenética do Centro Hospitalar São João, Portugal
}

\author{
Investigation of Genetic Aetiology in Neurodegenerative \\ Ataxias: Recommendations from the Group of \\ Neurogenetics of Centro Hospitalar São João, Portugal
}

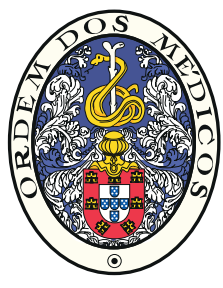

\author{
Tiago GOMES $\square^{1,2}$, Joana GUIMARÃES ${ }^{1,3}$, Miguel LEÃO ${ }^{4,5}$, em nome do Grupo de Neurogenética do Centro Hospitalar \\ São João \\ (Carla MOURA ${ }^{*, 5,6}$, Carolina GARRETT ${ }^{*, 1,3}$, Elsa AZEVEDO*,1,3, João MASSANO ${ }^{*, 3,7}$, João Paulo OLIVEIRA*,5,8, \\ Pedro CASTRO*,1,3) \\ Acta Med Port 2017 Jun;30(6):502-512 - https://doi.org/10.20344/amp.8797
}

\section{RESUMO}

Nas últimas décadas foi identificada uma lista crescente de ataxias neurodegenerativas de causa monogénica, permitindo uma melhor caracterização da patofisiologia, fenótipo e prognóstico deste grupo heterogéneos de patologias e revelando potenciais novos alvos terapêuticos. No entanto, a heterogeneidade e complexidade da relação genótipo-fenótipo e os elevados custos inerentes às técnicas de genética molecular, dificultam o plano racional clínico na orientação da investigação destas doenças. A história clínica é essencial à orientação diagnóstica, mas frequentemente o fenótipo não detém especificidade suficiente para permitir prever o genótipo. O Grupo de Neurogenética do Centro Hospitalar São João, grupo multidisciplinar de neurologistas e geneticistas com interesse especial na área das doenças neurogenéticas, delineou recomendações de consenso para a investigação da etiologia genética das ataxias neurodegenerativas, tendo por base documentos de consenso internacionais (contendo atualmente informação potencialmente desatualizada) e a evidência científica publicada sobre este tópico. Atualmente estão bem descritos aproximadamente 10 loci autossómicos recessivos e mais de 27 loci autossómicos dominantes para as ataxias neurodegenerativas. Este documento aborda de forma pragmática o processo utilizado para o diagnóstico genético das ataxias neurodegenerativas, com recomendações específicas para os diversos grupos de ataxias hereditárias e adaptado à realidade Portuguesa.

Palavras-chave: Ataxia/genética; Ataxia Espinocerebelosa/genética; Degeneração Espinocerebelosa/genética; Portugal

\section{ABSTRACT}

In recent decades, a long and increasing list of monogenic neurodegenerative ataxias has been identified, allowing for better characterization of the pathophysiology, phenotype and prognosis of this heterogeneous group of disorders, while also revealing potential new therapeutic targets. However, the heterogeneity and complexity of the genotype-phenotype relationships and the high costs of molecular genetics often make it difficult for clinicians to decide on a molecular investigation based on an unbiased rational plan. Clinical history is essential to guide the diagnostic workup, but often the phenotype does not hold enough specificity to allow for predicting the genotype. The Group of Neurogenetics of the Centro Hospitalar São João, a multidisciplinary team of neurologists and geneticists with special interest in neurogenetic disorders, devised consensus recommendations for the investigation of the genetic aetiology of neurodegenerative ataxias in clinical practice, based on international consensus documents (currently containing potentially outdated information) and published scientific evidence on this topic. At the time these recommendations were written, there were around 10 well described autosomal recessive loci and more than 27 autosomal dominant loci for neurodegenerative ataxias. This document covers, in a pragmatic way, the rational process used for the genetic diagnosis of neurodegenerative ataxias, with specific recommendations for the various groups of these heterogeneous diseases, per the Portuguese reality.

Keywords: Ataxia/genetics; Portugal; Spinocerebellar Ataxias/genetics; Spinocerebellar Degenerations/genetics

\section{INTRODUÇÃO}

O termo 'ataxia', do grego 'falta de ordem' alude à descoordenação e desorganização dos movimentos voluntários, que constituem este sinal neurológico, independentemente da força muscular. Isoladamente a ataxia é um sinal pouco específico e mau localizador e pode resultar da lesão ou disfunção de vários sistemas e níveis neuroló- gicos envolvidos na coordenação do movimento. Do ponto de vista semiológico é frequentemente dividida em ataxia cerebelosa ou sensitiva e a sua correta caracterização depende da história clínica e restantes achados do exame físico. As causas mais frequentes de ataxia são agudas ou subagudas e maioritariamente adquiridas (p.e. doença

\footnotetext{
1. Serviço de Neurologia. Centro Hospitalar São João. Porto. Portugal.

2. Departamento de Bioquímica. Faculdade de Medicina. Universidade do Porto. Porto. Portugal.

3. Departamento de Neurociências Clínicas e Saúde Mental. Faculdade de Medicina. Universidade do Porto. Porto. Portugal.

4. Unidade de Neuropediatria. Serviço de Pediatria. Centro Hospitalar São João. Porto. Portugal.

5. Serviço de Genética. Faculdade de Medicina. Universidade do Porto. Porto. Portugal.

6. Serviço de Otorrinolaringologia. Centro Hospitalar São João. Porto. Portugal.

7. Serviço de Neurologia. Hospital Pedro Hispano. Unidade Local de Saúde de Matosinhos. Matosinhos. Portugal.

8. Serviço de Nefrologia. Centro Hospitalar São João. Porto. Portugal.

* Colaboradores e membros do Grupo de Neurogenética do Centro Hospitalar São João, listados por ordem alfabética e afiliações respetivas.

$\triangle$ Autor correspondente: Tiago Gomes. tiago.bernardino.gomes@gmail.com

Recebido: 06 de fevereiro de 2017 - Aceite: 15 de maio de 2017 | Copyright @ Ordem dos Médicos 2017
} 
cerebrovascular, tóxicos, neuropatias e doenças neurodegenerativas adquiridas envolvendo as vias cerebelosas...), sendo o diagnóstico relativamente simples neste contexto. Existe um grupo de doenças neurodegenerativas mais raras e de causa genética ou presumivelmente genética em que a ataxia é um componente predominante do fenótipo, sendo por isso genericamente designadas de ataxias hereditárias $(\mathrm{AH})$.

As ataxias hereditárias são um grupo heterogéneo de doenças neurodegenerativas que se caracteriza fenotipicamente pela presença variável, mas significativa de algum tipo de ataxia, associada a uma diversidade muito variável de outros sinais neurológicos e sistémicos. Apesar de parte essencial do fenótipo, a ataxia é particularmente inespecíficas neste grupo patológico, sendo normalmente simétrica, mas de severidade, localização, início, progressão e tipo muito variáveis e com fraca correlação genótipo-fenótipo. Desta forma, representa uma designação sindrómica sem implicações etiológicas, dependendo o raciocínio clínico dos detalhes da história clínica e restantes sinais neurológicos que compõem o fenótipo completo.

Atualmente estão descritos mais de 10 loci autossómicos recessivos e mais de 27 loci autossómicos dominantes nos quais diversas mutações diferentes se podem associar ao desenvolvimento das ataxias hereditárias autossómicas dominantes (AH-AD) e ataxias hereditárias autossómicas recessivas (AH-AR), respetivamente. Existem ainda várias entidades clínicas sem defeito genético conhecido. Esta divisão, assim como a divisão em ataxias cerebelosas e sensitivas, representa uma convenção artificial, existindo uma elevada sobreposição fenotípica entre genótipos e diversas situações de formas alélicas em que mutações no mesmo gene produzem fenótipos distintos, incluindo outras patologias neurológicas como as paraparésias espásticas e as neuropatias hereditárias. ${ }^{1}$ Entre $1994-2004$, 39.7\% dos doentes com diagnóstico clínico de ataxia presumivelmente genética não tinha diagnóstico genético apesar de uma investigação extensa. ${ }^{2}$

A prevalência das ataxias hereditárias é muito variável entre países e depende, entre outros fatores, do fundo genético das populações. Em todo o mundo, a prevalências das $A H-A D$ varia entre 0,0 e $5,6 / 10^{5}$ e a das $A H-A R$ entre 0,0 e $7,2 / 10^{5}$, sendo que a prevalência global varia entre 1,3 e $20,2 / 10^{5}$ habitantes. ${ }^{1}$ Em Portugal, a prevalência das $\mathrm{AH}$ está calculada em $8,9 / 10^{5}$, sendo de 5,6 e 3,3/10 5 para as AH-AD e AH-AR, respetivamente. ${ }^{2}$ Entre 1994 - 2004, a ataxia espinocerebelosa tipo 3 (SCA3) era a mais prevalente das $\mathrm{AH}-\mathrm{AD}$ com $3,1 / 10^{5}$ e a ataxia de Friedreich a mais prevalente das $\mathrm{AH}-\mathrm{AR}$ com $1,0 / 10^{5}$ habitantes, sendo as ataxias com apraxia oculomotora o terceiro grupo mais prevalente com $0,4 / 10^{5}$ habitantes. $^{2}$

\section{Metodologia para elaboração do protocolo}

O Grupo de Neurogenética (GNgen) do Centro Hospitalar São João (CHSJ), formalizado em 2011 com a criação da Consulta de Grupo, reúne periodicamente para promover a partilha de conhecimento e a discussão clínica e cien- tífica no âmbito das doenças neurogenéticas. É constituído por um conjunto definido de profissionais provenientes de diferentes áreas técnico-científicas (lista de membros no final do artigo). As reuniões são abertas a todos os médicos do CHSJ que nelas queiram participar, nomeadamente os que têm interesse particular nestas doenças ou na Genética Médica, e médicos em formação.

A elaboração do protocolo de estudo genético nas ataxias neurodegenerativas foi entregue a um núcleo de autores que estabeleceram a metodologia de trabalho, pesquisaram e analisaram a literatura, coligiram os dados disponíveis e elaboraram uma primeira versão do protocolo, que foi enviada a todos os membros do GNgen por correio eletrónico. As várias sugestões obtidas por via eletrónica foram debatidas e incorporadas na versão definitiva que foi apresentada, debatida e aprovada em reunião do GNgen.

\section{ESTUDO GENÉTICO NAS ATAXIAS NEURODEGENE- RATIVAS: RECOMENDAÇÕES CLÍNICAS}

Este protocolo foi elaborado com base nas linhas de orientação emitidas conjuntamente pela European Federation of Neurological Societies (EFNS) e pela European Neurological Society (ENS), publicadas em 2014 no European Journal of Neurology. ${ }^{3}$ Estas recomendações são uma atualização das recomendações prévias provenientes da EFNS e publicadas em $2010,{ }^{4}$ pelo que estas foram também mantidas neste protocolo, dado que não são mutuamente exclusivas. Sempre que possível as recomendações foram adaptadas à realidade portuguesa com base em evidência científica publicada sobre este tópico ${ }^{1,2}$ e tendo em consideração o custo e acessibilidade dos painéis genéticos atualmente disponíveis para o estudo destas doenças. Os níveis de evidência apontados para cada recomendação são os utilizados de forma padronizada a nível internacional; sempre que não existam dados de Medicina Baseada na Evidência os peritos envolvidos na elaboração destas linhas de orientação emitiram uma recomendação consensual baseada na sua própria experiência e conhecimentos ('boa prática clínica'); os artigos originais estão facilmente acessíveis, na world wide web, para a consulta de mais detalhes.

Na maioria das situações o diagnóstico genético molecular será realizado em doentes com história familiar claramente consistente com uma forma monogénica da doença e de acordo com o padrão familiar de hereditariedade (autossómica dominante, recessiva ou ligada ao cromossoma X). O diagnóstico genético molecular poderá ainda ser aplicado em casos esporádicos com elevada suspeita clínica, dependendo das circunstâncias clínicas e da vontade dos envolvidos, após o devido esclarecimento detalhado.

\section{A classificação das ataxias de causa genética adotada neste documento será a seguinte:}

Ataxias espinocerebelosas autossómicas dominantes; Ataxias episódicas; Ataxias cerebelosas autossómicas recessivas; Ataxias ligadas ao cromossoma X; Ataxia crónica esporádica (quando suspeitar de causa genética). 


\section{I - Ataxias espinocerebelosas autossómicas dominan- tes}

Nas ataxias espinocerebelosas autossómicas dominantes ou SCA (spinocerebellar ataxias), a ataxia é frequentemente precedida, acompanhada ou sucedida por manifestações não cerebelosas como: neuropatia periférica, piramidalismo, parkinsonismo, coreia, distonia, mioclonias, demência, oftalmoplegia, surdez, difusão cognitiva, entre as mais frequentes. ${ }^{5}$ Até ao momento foram identificados mais de 27 loci genéticos para as SCAs salientando-se a SCA1-8, SCA10-23, SCA25-30, e a atrofia dentato-rubro-palido-luysiana (DRPLA), também incluída neste grupo. ${ }^{5}$

Existem três grupos major de SCA de acordo com a biologia molecular: (1) DRPLA e as SCA 1-3, 6, 7, 12, 17,

Tabela 1 - Ataxias espinocerebelosas autossómicas dominantes

\begin{tabular}{|c|c|c|c|c|}
\hline Nome & Locus/ Gene & Início (anos) & Duração (anos) & Proteínas/Mutação \\
\hline SCA1 & $\begin{array}{c}\text { 6p23 } \\
\text { ATXN1 }\end{array}$ & $\begin{array}{c}30-40 s \\
(<10 a>60)\end{array}$ & $\begin{array}{c}15 \\
(10-28)\end{array}$ & $\begin{array}{c}\text { Ataxina } 1 \text { / expansão CAG } 41-81 \\
\text { (normal } 25-36 \text { ) }\end{array}$ \\
\hline SCA2 & $\begin{array}{c}12 q 24 \\
\text { ATXN2 }\end{array}$ & $\begin{array}{c}30-40 s \\
(<10 a>60)\end{array}$ & $\begin{array}{c}10 \\
(1-30)\end{array}$ & $\begin{array}{c}\text { Ataxina } 2 \text { / expansão CAG } 35-59 \\
\text { (normal } 15-24)\end{array}$ \\
\hline $\begin{array}{l}\text { SCA3 } \\
\text { (MJD) }\end{array}$ & $\begin{array}{l}14 q 24.3-q 31 \\
\text { ATXN3 }\end{array}$ & $\begin{array}{l}4^{a} \text { década } \\
(10 \text { a } 70)\end{array}$ & $\begin{array}{c}10 \\
(1-20)\end{array}$ & $\begin{array}{c}\text { Ataxina } 3 \text { / expansão CAG } 62-82 \\
\text { (normal } 13-36)\end{array}$ \\
\hline SCA4 & $\begin{array}{c}16 q 22.1 \\
\text { diferente da SCA31 }\end{array}$ & $\begin{array}{l}4^{\mathrm{a}}-7^{\mathrm{a}} \text { década } \\
(19 \text { a } 72)\end{array}$ & Décadas & NA \\
\hline SCA5 & $\begin{array}{c}11 \mathrm{q} 13 \\
\text { SPTBN2 }\end{array}$ & $\begin{array}{l}3^{a}-4^{a} \text { década } \\
\quad(10 \text { a } 68)\end{array}$ & $>25$ & Espectrina $\beta$-III \\
\hline SCA6 & $\begin{array}{l}\text { 19p13.2 } \\
\text { CACNA1A }\end{array}$ & $\begin{array}{l}5^{a}-6^{a} \text { década } \\
(19 \text { a } 71)\end{array}$ & $>25$ & $\begin{array}{c}\text { Cav2.1 / expansão } \\
\text { CAG } 21-30 \text { (normal } 6-17)\end{array}$ \\
\hline SCA7 & $\begin{array}{l}\text { 3p21.1-p12 } \\
\text { ATXN7 }\end{array}$ & $\begin{array}{c}3^{a}-4^{a} \text { década } \\
(0,5 \text { a } 60)\end{array}$ & $\begin{array}{c}20 \\
(1-45)\end{array}$ & $\begin{array}{c}\text { Ataxina } 7 \text { / expansão CAG } 38-130 \\
\text { (normal } 7-17)\end{array}$ \\
\hline SCA8 & $\begin{array}{c}13 q 21.33 \\
\text { ATXN8OS/ ATXN8 }\end{array}$ & $\begin{array}{c}4^{\mathrm{a}} \text { década } \\
(1 \text { a } 64)\end{array}$ & Longevidade normal & $\begin{array}{l}\text { Não codificante/ } \\
\text { RNA tóxico }\end{array}$ \\
\hline SCA10 & $\begin{array}{l}22 q 13.31 \\
\text { ATXN10 }\end{array}$ & $\begin{array}{l}4^{\mathrm{a}} \text { década } \\
(12 \text { a } 48)\end{array}$ & 9 & $\begin{array}{l}\text { Expansão ATTCT } \\
\text { intrónica }\end{array}$ \\
\hline SCA11 & $\begin{array}{l}15 \mathrm{q} 15.2 \\
\text { TTBK2 }\end{array}$ & $\begin{array}{c}30 s \\
(15 \text { a } 70)\end{array}$ & Longevidade normal & $\begin{array}{l}\text { Cínase-2 da } \\
\text { Tau tubulina }\end{array}$ \\
\hline SCA12 & $\begin{array}{l}5 q 32 \\
\text { PPP2R2B }\end{array}$ & $\begin{array}{l}4^{\mathrm{a}} \text { década } \\
(8 \text { a } 62)\end{array}$ & NA & $\begin{array}{l}\text { PP2A / expansão CAG 51-78 } \\
\text { (normal } 7 \text { - 32) }\end{array}$ \\
\hline SCA13 & $\begin{array}{l}\text { 19q13.3-13.4 } \\
\text { KCNC3 }\end{array}$ & $\begin{array}{l}\text { Crianças } \\
\text { e adultos }\end{array}$ & NA & Kv3.3 \\
\hline SCA14 & $\begin{array}{l}19 q 13.4 \\
\text { PRKCG }\end{array}$ & $\begin{array}{c}3^{a}-4^{a} \text { década } \\
(3 \text { a } 70)\end{array}$ & $\begin{array}{l}\text { Décadas } \\
(1-30)\end{array}$ & PKCY \\
\hline SCA15 & $\begin{array}{l}\text { 3p26.1 } \\
\text { ITPR1 }\end{array}$ & $\begin{array}{c}4^{\mathrm{a}} \text { década } \\
(7 \text { a } 66)\end{array}$ & Décadas & ITPR \\
\hline $\begin{array}{l}\text { SCA17I } \\
\text { HDL4 }\end{array}$ & $\begin{array}{l}6 q 27 \\
\text { TBP }\end{array}$ & $\begin{array}{l}4^{\mathrm{a}} \text { década } \\
(3 \text { a } 55)\end{array}$ & $>8$ & $\begin{array}{l}\text { TBP / expansão CAG } 46 \text { - } 63 \\
\text { (normal } 25 \text { - 42) }\end{array}$ \\
\hline $\begin{array}{l}\text { SCA18/ } \\
\text { SMNA }\end{array}$ & $7 q 22-q 32$ & $(12-25)$ & Décadas & NA \\
\hline $\begin{array}{l}\text { SCA19/ } \\
\text { SCA22 }\end{array}$ & $\begin{array}{r}1 \mathrm{p} 13.3 \\
\text { KCND3 }\end{array}$ & $\begin{array}{l}4^{\mathrm{a}} \text { década } \\
(10 \text { a } 51)\end{array}$ & Décadas & Kv4.3 \\
\hline SCA23 & $\begin{array}{l}\text { 20p13 } \\
\text { PDYN }\end{array}$ & $\begin{array}{l}5^{a}-6^{a} \\
\text { década }\end{array}$ & $>10$ & Prodinorfina \\
\hline SCA27 & $\begin{array}{l}13 q 34 \\
\text { FGF14 }\end{array}$ & $\begin{array}{c}11 \\
(7-20)\end{array}$ & Décadas & FGF14 \\
\hline SCA28 & $\begin{array}{l}\text { 18p11.22-q11.2 } \\
\text { AFG3L2 }\end{array}$ & $\begin{array}{c}19,5 \\
(12-36)\end{array}$ & Décadas & Família das ATPases \\
\hline SCA31 & $\begin{array}{l}16 q 22 \\
\text { BEAN1 }\end{array}$ & $\begin{array}{l}5^{a}-6^{a} \\
\text { década }\end{array}$ & Longevidade normal & $\begin{array}{l}\text { Expansão TGGAA } \\
\text { intrónica }\end{array}$ \\
\hline SCA35 & $\begin{array}{l}20 p 13 \\
\text { TGM6 }\end{array}$ & $\begin{array}{c}43,7 \pm 2,9 \\
(40-48)\end{array}$ & $\begin{array}{l}15,9 \pm 8,8 \\
(5-31)\end{array}$ & Transglutaminase 6 \\
\hline SCA36 & $\begin{array}{l}20 p 13 \\
\text { NOP56 }\end{array}$ & $5,2,8 \pm 4,3$ & Décadas & $\begin{array}{c}\text { Expansão GGCCTG } \\
\text { intrónica }\end{array}$ \\
\hline DRPLA & $\begin{array}{l}\text { 12p13.31 } \\
\text { ATN1 }\end{array}$ & $\begin{array}{c}3^{a}-4^{a} \text { décadas } \\
(8-20 \text { ou } 40-60)\end{array}$ & $\begin{array}{l}\text { Início precoce associa-se com } \\
\text { menor longevidade }\end{array}$ & $\begin{array}{l}\text { Atrofina } 1 \text { / expansão CAG } 49-75 \\
\text { (normal } 7-23)\end{array}$ \\
\hline
\end{tabular}


causadas pela expansão de CAG na região de codificação (também chamadas ataxias de poli-glutamina), constituindo este tipo de mutação a causa mais comum de ataxias autossómicas dominantes; (2) SCAs 8, 10, 31 e 36 resultantes de expansões repetidas em regiões não codificantes; (3) SCA 5, 11, 13, 14, 15/16, 19/22 e 27, causadas por outros tipos de mutações que não expansões (p.e. pontuais) ${ }^{6}$
A SCA6 pode ser causada por ambos os tipos de mutação, mas sobretudo expansões. ${ }^{6} \mathrm{O}$ estudo molecular deve ser dirigido de acordo com os sinais ou sintomas associados à ataxia (Tabela 1) e de acordo com a origem geográfica do caso índex.

Na Europa, de acordo com a sua prevalência, as SCA podem subdividir-se em 4 subgrupos:1-3

Tabela 1 - Ataxias espinocerebelosas autossómicas dominantes (secção final)

\section{Fenótipo distintivo}

Sinais piramidais, neuropatia periférica

Sacadas lentas, neuropatia periférica, hiporreflexia, demência (rara)

Sinais piramidais e extrapiramidais, retração palpebral, nistagmo, sacadas lentas, amiotrofia com fasciculações e défice sensitivo.

Neuropatia axonal sensitiva e surdez

Progressão lenta com síndrome piramidal (precoce) ou ataxia cerebelosa pura (tardia)

Início tardio (> 50 anos), progressão muito lenta e ataxia cerebelosa pura (às vezes episódica)

Hipovisão com retinopatia (degenerescência pigmentar da mácula), oftalmoplegia externa e sinais piramidais

Lentamente progressiva com sinais piramidais, hipostesia vibratória e demência (raro)

Crises ocasionais (nativos americanos)

Ataxia cerebelosa pura ligeira (mantêm marcha)

Lentamente progressiva e ligeira com tremor de ação aos $30 \mathrm{~s}$, sinais piramidais e parkinsonismo subtis, distúrbio neuropsiquiátrico incluindo demência.

Baixa estatura e atraso cognitivo ligeiro (pedigree Francês) e ataxia cerebelosa pura (Filipino)

Mioclonias axiais precoces com ataxia tendencialmente pura

Ataxia cerebelosa pura lentamente progressiva.

Demência; coreia, distonia e mioclonias ocasionais; epilepsia.

Ataxia com neuropatia SM precoce, nistagmo, disartria, hiporreflexia, tetraparésia, atrofia e fasciculações e Babinski.

Lentamente progressiva e raramente com mioclonias, hiperreflexia e demência.

Tardia (> 50 anos) com disartria, alteração da oculomotricidade, disfunção da sensibilidade vibratória e posicional.

Tremor precoce, discinesias orofaciais e demência.

Nistagmo, oftalmoplegia externa, ptose, hiperreflexia.

Ataxia pura tardia (> 50 anos) e surdez neurossensorial.

Sinais piramidais e torcicolos espasmódico.

Sinais de $2^{\circ}$ neurónio motor com atrofia da língua.

Coreia, epilepsia, demência e mioclonias.

Adaptada de Gasser et al. ${ }^{2}$ SCA: Ataxia espinocerebelosa; ATXN 1/2/3/7/8/10: Ataxina 1/2/3/7/8/10; CACNA1A: Subunidade alfa-1a do canal de cálcio; DRPLA: Atrofia dentato-rubro-palidoluisiana; FGF14: Fator de crescimento do fibroblasto 14; ITPR1: Recetor do inositol 1,4,5-trifosfato tipo 1; KCNC3: Canal de potássico 3; PPP2R2B: Subunidade B da fosfatase 2 de proteínas; TBP: Proteína de ligação TATA Box; TTBK2: Cínase 2 da Tau tubulina. 
- SCA3;

- SCA2, SCA6;

- SCA1, SCA7, SCA8:

- SCA10, SCA12, SCA17.

A idade de início mais comum encontra-se entre os trinta e os cinquenta anos, mas muitas foram já documentadas em crianças e/ou idades mais avançadas, sendo que a idade de início e a severidade do quadro são frequentemente relacionadas com o aumento do número de repetições nas mutações por expansão, fenómeno de antecipação (p. ex. SCA2 e a SCA7). ${ }^{3,5-7}$ No entanto, o início na infância e progressão muito lenta podem orientar o diagnóstico genético e foram descritas em casos de SCA13 e 14.,35-7 A anamnese e exame neurológico fornecem estes e outros dados que ajudam na estratificação do estudo molecular.

A SCA3 é a mais prevalente em Portugal $\left(3,2 / 10^{5}\right)$, mas também a mais heterogénea, enquanto as SCA4, SCA18, SCA19/22, SCA21, SCA25, SCA26, SCA28 e SCA30 são consideradas as mais 'puras', especialmente na fase inicial da doença. ${ }^{5}$ Algumas manifestam-se frequentemente com sintomas não cerebelosos como a SCA7 com disfunção visual (49\%), a SCA14 com mioclonias ( $8 \%$ ), a SCA17 com parkinsonismo (12\%), alterações psiquiátricas (19\%) ou disfunção cognitiva (13\%); a SCA13 com atraso de desenvolvimento psicomotor (61\%) e a DRPLA com disfunção cognitiva (15\%), atraso de desenvolvimento psicomotor (7\%) e alterações psiquiátricas (4\%). ${ }^{5}$ A DRPLA é incomum na Europa, mas deve ser considerada no caso de ascendência Asiática e é relativamente mais frequente em Portugal $\left(0,33 / 10^{5}\right.$ entre 1994 - 2004) comparando com o resto da Europa. ${ }^{2}$ Para um resumo completo das SCA ver Tabela 1.

\section{Recomendações para o estudo genético das ataxias espinocerebelosas autossómicas dominantes}

Tendo em conta as recomendações europeias relativas ao estudo molecular genético nas $\mathrm{SCA},{ }^{3}$ a respetiva epidemiologia ${ }^{1,2}$ e a relação custo-benefício das plataformas de diagnóstico molecular comercialmente disponíveis, as recomendações para a realização de testes genéticos nos casos de AH com história familiar autossómica dominante, são as seguintes (recomendações de nível B de evidência presentes nos documentos originais da EFNS de $2010^{4} \mathrm{e}$ EAN/EFNS de $2014^{3}$ e adaptadas à realidade Portuguesa pelo GNgen do $\mathrm{CHSJ}^{1,2}$ ):

$1^{\text {a }}$ linha: SCA1, SCA2, SCA3, SCA6

$2^{\mathrm{a}}$ linha: SCA8, SCA10, SCA12, SCA17

$3^{\text {a }}$ linha: SCA7

$4^{a}$ linha: DRPLA (se ascendência asiática, maior prevalência em Portugal versus Europa) ${ }^{2}$

A priorização prévia carece de adaptação dependente do caso individual com base na história familiar (p.e. ascendência asiática) e nos detalhes do fenótipo (p.e. retinopatia precoce na SCA7) (recomendação de boa prática clínica pela EFNS $(2010)^{4}$ adaptada pelo GNgen do CHSJ).
1. Se o estudo genético inicial (referido em 1.) for negativo é recomendada a referenciação a um centro clínico especializado no diagnóstico neurogenético para revisão do fenótipo e orientação do estudo genético posterior (recomendação de boa prática clínica pela EAN/EFNS (2014) $)^{3}$ adotada pelo GNgen do CHSJ).

2. Existem algumas doenças heredodegenerativas autossómicas dominantes que podem cursar com disfunção cerebelosa significativa, nomeadamente ataxia. Apesar da história clínica e estudo imagiológico normalmente apresentarem características diferenciais, existem algumas doenças que devem ser tidas em consideração no diagnóstico diferencial das SCA, nomeadamente: as neuroferritinopatias, a atrofia ótica 1 (OPA1), doenças priónicas hereditárias, doença de Alexander e leucodistrofia e ataxia espástica do adulto 1 (SPAX1) (recomendação de boa prática clínica pela EAN/EFNS (2014) ${ }^{3}$ adotada pelo GNgen do CHSJ).

\section{II - Ataxias episódicas}

As ataxias episódicas (EA) caracterizam-se por episódios recorrentes de ataxia, perda de equilíbrio e vertigem. ${ }^{8}$ Estes episódios podem durar minutos (EA1) ou várias horas (EA2) podendo ser precipitadas por movimentos súbitos na EA1; pelo exercício, stresse emocional, álcool e cafeína na EA2. ${ }^{8}$ Nos intervalos entre os episódios pode existir mioquimia das mãos (detetada por EMG) no caso da EA1. Na EA2, os doentes podem apresentar, entre os episódios de ataxia, nistagmo em posições extremas do olhar. ${ }^{8}$ A incidência global das EA é $\sim 1 / 10^{5}$, mas estará provavelmente subestimada devido a exigência dos testes genéticos disponíveis e casos sem mutação identificada. ${ }^{8}$ As EA são maioritariamente canalopatias autossómicas dominantes envolvendo canais de $\mathrm{K}^{+}$, como o KCNA1 (EA1), e de $\mathrm{Ca}^{2+}$ como os CACNA1A (EA2) e CACNB4 (EA5)..$^{8} \mathrm{O}$ gene para o transportador de glutamato SLC1A3 causa a EA6. ${ }^{8}$ A EA2, a SCA6 e a enxaqueca hemiplégica familiar, são fenótipos distintos de mutações alélicas, podendo, em alguns indivíduos ou famílias, existir sobreposição fenotípi$\mathrm{ca}^{4}$

\section{Recomendações para o estudo genético das ataxias episódicas}

A realização de testes genéticos para a EA1 e EA2 está indicada em doentes com episódios recorrentes de ataxia e vertigem e com história familiar de transmissão autossómica dominante (recomendação da EFNS (2010) de nível C de evidência). ${ }^{4}$

\section{III - Ataxias cerebelosas autossómicas recessivas}

São um grupo muito heterogéneo de doenças neurodegenerativas, a maioria muito raras, que se caracterizam por ataxia cerebelosa precoce associada a outros sinais neurológicos, sistémicos e oftalmológicos. As características neurológicas mais importantes são: atrofia óptica, sinais piramidais e extrapiramidais, neuropatia periférica, disfunção cognitiva e epilepsia. ${ }^{9,10} \mathrm{Na}$ Europa as causas de $\mathrm{AH}$ 
autossómica recessiva mais prevalentes são a ataxia de Friedreich (FRDA), a ataxia telangiectásica (AT) e as ataxias com apraxia oculomotora (AOA). ${ }^{3}$ Em Portugal, entre 1994 - 2004, a FRDA era a mais prevalente $1,0 / 10^{5}$, seguindo-se a AOA com $0,40 / 10^{5}\left(0,13 / 10^{5}\right.$ para a AOA1) e finalmente a AT com $0,15 / 10^{5}$ habitantes. ${ }^{2}$ De seguida descrevem-se as principais ataxias cerebelosas autossómicas recessivas, para o resumo detalhado das mesmas ver Tabela 2.

\section{Ataxia de Friedreich (FRDA)}

Apresenta-se como ataxia axial e dos membros, habitualmente antes dos 25 anos, e progride com disartria, hiporreflexia/arreflexia, hipostesia e défice motor, existindo cardiomiopatia na maioria dos doentes. ${ }^{7,9}$ A neuropatia sensitiva axonal, défice motor de predomínio distal, escoliose, surdez neurossensorial, atrofia óptica e diabetes são características comuns a muitos fenótipos. ${ }^{7,9}$ A doença é causada pela expansão do trinucleotídeo GAA no primeiro intrão do gene FRDA no cromossoma 9q13-21 e o tamanho da expansão é inversamente correlacionado com a idade de início da doença, grau de incapacidade e incidência de cardiomiopatia..$^{7,9}$

\section{Ataxia com apraxia oculomotora (AOA)}

Caracteriza-se por ataxia, apraxia oculomotora e movimentos coreoatetósicos, podendo apresentar-se sob 4 formas geneticamente distintas AOA1-4. ${ }^{7}$ A AOA1 caracteriza-se por ataxia cerebelosa, neuropatia sensitivo-motora, nistagmo, apraxia oculomotora variável, sinais extrapiramidais e disfunção cognitiva moderada, com início no final da primeira década de vida ou início da segunda. ${ }^{7}$ Os doentes podem apresentar hipoalbuminemia ou hipercolesterolemia, sendo a alfa-fetoproteína normal. ${ }^{7}$ A AOA2 apresenta um fenótipo semelhante, mas a idade de início é mais tardia (por volta dos 20s); os valores de albumina são normais e existe elevação da alfa-fetoproteína. ${ }^{7}$ AAOA3 é uma doença rara com início na segunda década de vida e fenótipo semelhante às restantes. ${ }^{7}$ A AOA4 caracteriza-se por início na primeira década de vida com distonia e ataxia, sendo a apraxia oculomotora e neuropatia periférica características adicionais, com alguns doentes a desenvolver disfunção cognitiva. ${ }^{7}$ A AOA4 é progressiva e a maior parte dos doentes perde a marcha autónoma entre a segunda e terceira décadas de vida. ${ }^{7}$ Em Portugal, as formas mais frequentes são a $\mathrm{AOA} 1^{2}$ e possivelmente a $\mathrm{AOA} 4{ }^{11}$.

\section{Ataxia telangiectásica (AT) $)^{7,9}$}

A AT caracteriza-se por ataxia cerebelosa, apraxia oculomotora, telangiectasias, alterações imunológicas e predisposição para malignidade. A ataxia inicia-se, em regra, antes dos cinco anos de idade, com posterior desenvolvimento de telangiectasias. Podem ocorrer movimentos coreoatetósicos em $90 \%$ dos doentes e a elevação de alfa-fetoproteína é típica. O risco de desenvolvimento de neoplasias é cerca de $40 \%$, sendo as mais frequentes os linfomas e as leucemias.

\section{Ataxia com défice de vitamina $E$ (ADVE) e abeta-lipo- proteinemia (ABLP) $)^{7,9}$}

A ADVE apresenta fenótipo semelhante à ataxia de Friedreich, com idade de início antes dos 20 anos e, tipicamente, com níveis séricos baixos de vitamina E. Alguns doentes apresentam retinite pigmentar com perda da acuidade visual. A maior parte das séries descritas tem origem na bacia mediterrânica. A ABLP apresenta um fenótipo neurológico semelhante à ataxia com défice de vitamina $E$, mas está associada à má absorção lipídica, hipocolesterolemia e acantocitose.

\section{Recomendações para o estudo genético das ataxias cerebelosas autossómicas recessivas}

1. Tendo em conta as recomendações europeias relativas ao estudo molecular genético nas $\mathrm{AH}$ autossómicas recessivas $^{3}$, a respetiva epidemiologia ${ }^{1,2,9,12}$ e a relação custo-benefício das plataformas de diagnóstico molecular comercialmente disponíveis, as recomendações para a realização de testes genéticos nos casos de AH com história familiar autossómica recessiva, são as seguintes:

1.1) A FRDA deve ser sempre testada em casos de ataxia isolada e ausência de história familiar, independentemente da idade e mesmo nos casos com reflexos osteotendinosos preservados (recomendações de nível B de evidência da EFNS (2010) 4 e boa prática clínica da EAN/EFNS $(2014)^{3}$ adaptadas à realidade Portuguesa pelo GNgen do $\mathrm{CHSJ}^{1,2}$;

1.2) Previamente à realização de estudos de genética molecular devem ser realizados os seguintes exames complementares de diagnóstico: RM de neuro-eixo, doseamento de vitamina $\mathrm{E}$, colestenol e colesterol, albumina, cínase da creatina, alfa-fetoproteína, anticorpos anti-GAD e anti-tiroideus. Considerar ECN/EMG para avaliar presença e tipo de neuropatia associada. Observação por Oftalmologia para avaliar a presença de cataratas, retinite pigmentosa, mancha vermelha da mácula ou outras (recomendações de nível B de evidência da EFNS (2010) 4 e boa prática clínica da EAN/EFNS (2014) ${ }^{3}$ adotadas pelo GNgen do CHSJ);

1.3) Considerar ainda estudo de enzimas leucocitárias e doseamento sérico de ácido fitânico e ácidos gordos de cadeia longa (recomendações de nível $B$ de evidência da EFNS $(2010)^{4}$ e boa prática clínica da EAN/EFNS (2014) adotadas pelo GNgen do CHSJ).

2. Em função do fenótipo e resultados dos exames complementares de diagnósticos sugeridos nas recomendações prévias, hierarquizar o estudo molecular da seguinte forma (recomendações de boa prática clínica do GNgen do CHSJ após avaliação da literatura) $)^{1-4}$ :

\author{
A. Se > 10 anos de idade: \\ $1^{\text {a }}$ linha: FRDA \\ $2^{a}$ linha: AOA1, AOA4 \\ $3^{a}$ linha: AOA2, POLG \\ $4^{\mathrm{a}}$ linha: ABLP, ADVE, SACS, SPG7, AT
}

\section{B. Se $<10$ anos de idade: $1^{\mathrm{a}}$ linha: AOA1, AOA4}


$2^{\text {a }}$ linha: FRDA, ADVE

$3^{\mathrm{a}}$ linha: AOA2

Se $<2$ anos de idade deve ser testada a AT.

Em última análise poderá estar indicada biopsia de pele e/ou músculo para o diagnóstico de doenças de Niemann-Pick tipo $C$, ataxia recessiva com défice de coenzima $Q$ ou ponderar a hipótese de mitocondriopatia (recomendação de boa prática clínica da EAN/EFNS (2014) ${ }^{3}$ adaptada pelo GNgen do CHSJ).

Tabela 2 - Ataxias cerebelosas autossómicas recessivas

\begin{tabular}{|c|c|c|c|}
\hline Nome & Gene & Início (anos) & Estudo sugerido \\
\hline Ataxia de Friedreich & FRDA & $\begin{array}{c}5-25 \\
\text { (tardio comum) }\end{array}$ & Estudo genético \\
\hline Ataxia hereditária com deficiência de vitamina $E$ & TTPA & $<20$ & Vit. E no sangue baixa/ estudo genético \\
\hline Ataxia espinocerebelosa infantil (IOSCA) & C10orf2 & $<2$ & Estudo genético \\
\hline Ataxia de Cayman & ATCAY & $\begin{array}{c}<20 \\
\text { (à nascença) }\end{array}$ & Estudo genético \\
\hline $\begin{array}{l}\text { Ataxia espinocerebelosa com neuropatia } \\
\text { axonal (SCAN) }\end{array}$ & TDP1 & $<20$ & $\begin{array}{c}\text { Hipercolesterolemia e hipoalbuminemia/ } \\
\text { estudo genético }\end{array}$ \\
\hline Doença de Refsum & PHYH / PEX7 & $\begin{array}{l}<20 \\
\text { (tardio possível) }\end{array}$ & $\begin{array}{c}\text { Acido fitânico elevado no sangue / } \\
\text { estudo genético }\end{array}$ \\
\hline Abeta-lipoproteinemia & MTP & $<20$ & $\begin{array}{l}\text { Acantócitos, hipocolesterolemia, vit. E } \\
\text { no sangue baixa / estudo genético }\end{array}$ \\
\hline Ataxia espástica AR de Charlevoix-Saguenay & SACS & $\begin{array}{l}\quad<10 \\
\text { (tardio possível) }\end{array}$ & Estudo genético \\
\hline Ataxia telangiectasia & ATM & $\begin{array}{l}2 \text { - } 3 \\
\text { (tardio possível) }\end{array}$ & $\begin{array}{c}\text { a-fetoproteína elevada no sangue / } \\
\text { estudo genético }\end{array}$ \\
\hline Ataxia telangiectasia-like & MRE11 & $<20$ & Estudo genético \\
\hline Ataxia com apraxia oculomotora tipo 1 (AOA1) & APTX & $\begin{array}{l}<20 \\
\text { (tardio possível) }\end{array}$ & $\begin{array}{c}\text { Hipercolesterolemia e hipoalbuminemia / } \\
\text { estudo genético }\end{array}$ \\
\hline Ataxia com apraxia oculomotora tipo 2 (AOA2) & SETX & $\begin{array}{l}\quad<20 \\
\text { (tardio possível) }\end{array}$ & $\begin{array}{l}\text { Hipercolesterolemia e a-fetoproteína } \\
\text { elevada no sangue }\end{array}$ \\
\hline Xantomatose cerebrotendinosa & CYP27 & $\begin{array}{l}<20 \\
\text { (tardio possível) }\end{array}$ & $\begin{array}{l}\text { Colestanol urinário elevado e } \\
\text { hipercolesterolemia / estudo genético }\end{array}$ \\
\hline Síndrome de Marinesco-Sjogren & SIL1 & Infância & CK elevada / estudo genético \\
\hline Doença de Tay-Sachs tardia & HEXA & $\begin{array}{c}<20 \\
\text { (tardio possível) }\end{array}$ & $\begin{array}{c}\text { Redução da atividade da } \\
\text { hexosaminidase } \mathrm{A} / \text { estudo genético }\end{array}$ \\
\hline $\begin{array}{l}\text { Ataxia cerebelosa AR (ARCA) } \\
\text { com atraso psicomotor }\end{array}$ & SYT14 & $50-56$ & Estudo genético \\
\hline $\begin{array}{l}\text { ARCA associada a espectrina tipo } 1 \\
\text { (SPARCA1) }\end{array}$ & SPTBN2 & Infância precoce & Estudo genético \\
\hline ARCA2 & ADCK3/CABC1 & $1-11$ & $\begin{array}{c}\text { Níveis baixos de } \\
\text { coenzima } Q_{10} \text { em biopsia de músculo }\end{array}$ \\
\hline $\begin{array}{l}\text { Ataxia sensitiva neuropática com disartria e } \\
\text { oftalmoparesia (SANDO) }\end{array}$ & POLG1 & $15-40$ & Estudo genético \\
\hline Ataxia cerebelosa AR tipo 1 (ARCA1) & SYNE1 & $17-50$ & Estudo genético \\
\hline ARCA3 & ANO10 & $13-45$ & EMG com DNM / estudo genético \\
\hline $\begin{array}{l}\text { Ataxia e anemia sideroblástica ligadas ao X } \\
\text { (XLSA/A) }\end{array}$ & $\mathrm{ABCB} 7$ & $2-4$ & $\begin{array}{c}\text { Hemograma completo e esfregaço } \\
\text { sanguíneo/ estudo genético }\end{array}$ \\
\hline
\end{tabular}

Adaptada de Gasser et al. ${ }^{2}$ ATM: Gene mutado na ataxia telangiectasia; MRE11: Recombinação meiótica 11; MTP: Proteína de transferência microssomal; SPTBN2: Espectrina beta não eritróide 2 . 
justifica-se a realização de estudo de genética molecular de AOA2;

D. Em doentes com ataxia e evidência de imunodeficiência (mais frequentemente de $\operatorname{lgA}$ e $\operatorname{lgG} 4$ ) com alfa-fetoproteína elevada justifica-se a realização de estudo de genética molecular de AT.

\section{IV - Ataxias ligadas ao cromossoma $X^{3,9}$}

Na síndrome da ataxia/tremor $X$ frágil os doentes apresentam pela sexta década de vida (invariavelmente acima dos 45 anos), tremor de intenção de agravamento progres- sivo, ataxia, parkinsonismo, deterioração cognitiva e neuropatia periférica. A presença de lesões hiperintensas em T2 nos pedúnculos cerebelosos e substância branca cerebelosa adjacente é típica desta entidade.

Recomendações para o estudo genético das AH ligadas ao cromossoma $X$

Está indicada a realização de pesquisa de FMR1 nos casos de ataxia e parkinsonismo com início pela $6^{\text {a }}$ década de vida ou antes, com história familiar compatível com hereditariedade ligada ao cromossoma $\mathrm{X}$ e

Tabela 2 - Ataxias cerebelosas autossómicas recessivas (secção final)

\section{Fenótipo}

Ataxia cerebelosa/sensitiva, arreflexia, s. piramidal (pes cavus) com sinal de Babinski, cardiomiopatia, diabetes, atrofia óptica e da espinal medula.

Semelhante à Friedreich, com hipovisão ou retinitis pigmentosa, espasticidade, coreia, cardiomiopatia e diabetes possíveis, atrofia cerebelosa variável.

Hipotonia, neuropatia, arreflexia, atrofia óptica, oftalmoplegia externa, surdez neurossensorial, movimentos involuntários, epilepsia, atrofia cerebelosa, hipersinal da substância branca e edema cortical.

Hipotonia, sinais cerebelosos mais tardios com nistagmo e atraso mental. Hipoplasia cerebelosa.

Neuropatia axonal sensitivo-motora, amiotrofia distal, pes cavus, atrofia cerebelosa. (Arábia Saudita).

Polineuropatia, surdez neurossensorial, retinitis pigmentosa, anosmia.

Semelhante à Friedreich, retinitis pigmentosa, má absorção intestinal.

Espasticidade, neuropatia severa, fibras do nervo óptico hipermielinizadas. Atrofia cerebelosa pode ser tardia.

Apraxia oculomotora, telangiectasias conjuntivais, sinais extrapiramidais, risco de neoplasia, atrofia cerebelosa.

Semelhante à AT, sem telangiectasias, com apraxia oculomotora e coreia, atrofia cerebelosa.

Semelhante à AT, neuropatia sensitivo-motora, coreia, disfunção cognitiva, atrofia cerebelosa.

Semelhante à AOA1, com menos apraxia e neuropatia moderada/ligeira, atrofia cerebelosa.

Sinais piramidais e extrapiramidais, convulsões, demência, cataratas juvenis, xantomas tendinosos

Miopatia, cataratas, deformidades esqueléticas, hipogonadismo, atraso cognitivo ligeiro.

Arreflexia, paresia e atrofia proximais, distúrbios comportamentais.

Atraso psicomotor e distúrbios dos movimentos de perseguição ocular.

Atraso cognitivo, esotropia e hipotonia.

Epilepsia, atraso cognitivo, atrofia cerebelosa.

Oftalmoplegia externa, neuropatia, mioclonias, distonia, encefalopatia.

Ataxia cerebelosa tendencialmente pura

Nistagmo downbeat, hiperreflexia

Sinais do primeiro neurónio nos membros inferiores de homens, dificuldades de aprendizagem ligeiras, depressão, anemia.

Adaptada de Gasser et al. ${ }^{2}$ ATM: Gene mutado na ataxia telangiectasia; MRE11: Recombinação meiótica 11; MTP: Proteína de transferência microssomal; SPTBN2: Espectrina beta não eritróide 2. 
recessiva, ou em casos isolados de homens afetados com ataxia/ parkinsonismo e história familiar em tias ou sobrinhas com amenorreia primária ou insuficiência ovárica prematura (menopausa < 40 anos) de etiologia não esclarecida (recomendações de nível B de evidência da EFNS $(2010)^{4}$ e boa prática clínica da EAN/EFNS $(2014)^{3}$ adotadas pelo GNgen do CHSJ).

\section{V - Ataxia crónica esporádica (quando suspeitar de causa genética) $)^{3,7,9,13}$}

Casos esporádicos de ataxias de causa genética não são incomuns, mas as causas adquiridas são mais frequentes, potencialmente tratáveis e exigem um estudo etiológico aprofundado. Por exemplo, ataxia e marcada disautonomia em fase precoce e acima dos 30 anos sugerem MSA (atrofia de múltiplos sistemas).

A neuroimagem é essencial à orientação do diagnóstico e poderá revelar alterações estruturais indicadoras da priorização do diagnóstico diferencial. O estudo analítico para causas adquiridas é essencial, particularmente na ausência de lesão estrutural sugestiva, nomeadamente: vitamina E, B1 e B12, TSH e T4 livre, cálcio, fosfato e anticorpos anti-GAD.

\section{Recomendações para o estudo genético na ataxia cró- nica esporádica}

1. Previamente à realização de estudos de genética molecular devem ser excluídas as causas adquiridas através de neuroimagem (cerebral e espinal medula) e estudo analítico de sangue; a hipótese de MSA deve ser sempre considerada se início depois dos 30 anos (até 29\% dos casos de ataxia esporádica sem outra causa adquirida reconhecível preenchem critérios de MSA provável ou possível) (recomendação de boa prática clínica da EAN/EFNS (2014) $)^{3}$ adotada pelo GNgen do CHSJ).

2. Não está recomendada a pesquisa da sensibilidade ao glúten em casos esporádicos de ataxia cerebelosa isolada (recomendação de nível B de evidência da EAN/EFNS (2014) $)^{3}$ adotada pelo GNgen do CHSJ).

3. No caso de exclusão de causas adquiridas, ou manutenção de dúvida razoável, há lugar para ponderar estudo genético, com base na possibilidade de ocultação de laços familiares, penetrância incompleta, mutações de novo ou morte precoce, nas formas de início mais tardio:

3.1. No caso de ataxia esporádica e independentemente da idade de início, recomenda-se estudo genético para SCA1-3, 6 e DRPLA (a última especialmente se ascendência asiática) (recomendação de nível $\mathrm{B}$ de evidência da EAN/EFNS (2014) ${ }^{3}$ adotada pelo GNgen do CHSJ);

3.2. Atualmente as ataxias recessivas devem também ser contempladas no adulto, nomeadamente o teste genético do FRDA, embora outros estudos bioquímicos (ex. colestanol, vitamina $\mathrm{E}$, albumina, CK e alfa-fetoproteína) devam também ser ponderados (recomendação de nível B de evidência da EAN/EFNS $(2014)^{3}$ adotada pelo GNgen do CHSJ);

3.3. Se o estudo prévio for negativo e a idade de início for abaixo dos 45 anos, recomenda-se seguir as recomendações completas referidas em (III. Ataxias cerebelosas autossómicas recessivas) (recomendação de boa prática clínica da EAN/EFNS (2014) ${ }^{3}$ adaptadas pelo GNgen do CHSJ à realidade Portuguesa $)^{1,2}$;

3.4. Se o estudo prévio for negativo e a idade de início for acima dos 45 anos, recomenda-se pesquisa da prémutação do FMR1 em homens (recomendação de nível B de evidência da EAN/EFNS $(2014)^{3}$ adotada pelo GNgen do CHSJ);

3.5. Na eventualidade de se julgar necessário prosseguir com a pesquisa de formas genéticas mais raras de ataxia, recomenda-se a discussão do caso clínico concreto em ambiente multidisciplinar especializado antes de prosseguir para outros eventuais testes laboratoriais (prática habitual do GNgen do CHSJ).

NOTAS (adaptadas dos documentos da EFNS $(2010)^{4}$ e EAN/EFNS (2014) $)^{3}$ pelo GNgen do CHSJ):

A. A ataxia anti-GAD tem frequentemente um início subagudo, mas pode ser consideravelmente insidiosa. $\mathrm{Na}$ presença de títulos altos no sangue, sugere-se avaliação do LCR para comprovar síntese intratecal, sendo que a sua ausência torna pouco provável o diagnóstico e sugere síndrome autoimune sistémico endócrino ( $p$ ex. diabetes tipo 1).

B. A degenerescência cerebelosa alcoólica é uma complicação frequente no abuso crónico, mas não existem dados sobre qual a dose e/ou duração de exposição necessárias para causar doença. A procura de doença hepática e neuropatia é importante, mas sugere-se o estudo completo e ponderar causa genética apenas no caso de dúvida razoável.

C. A ataxia por sensibilidade ao glúten é atualmente uma entidade controversa e sem tratamento eficaz comprovado. Os anticorpos encontrados apresentam a mesma prevalência entre os casos de ataxias genéticas comprovadas e ataxias esporádicas, sendo ainda frequentes na população geral, suspeitando-se que possa ser apenas um epifenómeno.

\section{NOTAS FINAIS}

As Tabelas 1 e 2 resumem, respetivamente, as características principais das várias ataxias cerebelosas autossómicas dominantes e autossómicas recessivas. Trata-se de uma lista exaustivas que, no entanto, não inclui as ataxias presumivelmente hereditárias sem diagnóstico genético definido ou entidades muito raras e ainda mal definidas.

Este protocolo será revisto sempre que seja entendido necessário e oportuno pelo GNgen do CHSJ.

\section{CONSIDERAÇÕES FINAIS}

Os avanços tecnológicos e laboratoriais, em especial o surgimento de novas técnicas de sequenciação genética mais rápidas, precisas, eficazes, eficientes e, acima de tudo potencialmente mais económicas, como a next generation sequencing e a whole exome sequencing, permitem 
agora pesquisar mutações num grande número de genes simultaneamente. ${ }^{7}$ Estas novas técnicas levaram à criação de kits e/ou sistemas comercialmente viáveis que agregam a pesquisa de diversos genes de interesse num só kit e permitem a sua realização num só tempo. Aliando o raciocínio científico à necessidade de gerir recursos económicos e logísticos têm vindo a ser desenvolvidos diversos kits, mais vulgarmente chamados "painéis" genéticos, para o teste de grupos de doenças hereditárias clinicamente relacionadas e quase sempre difíceis de distinguir pela elevada variabilidade ou sobreposição fenotípica. ${ }^{6,7,10}$ No caso das ataxias hereditárias existem já diversos painéis deste tipo comercialmente disponíveis, normalmente agregados de acordo com o tipo de hereditariedade. Em diversos grupos de doenças neurodegenerativas hereditárias o uso de painéis genéticos traz várias vantagens para a atividade assistencial e gestão económica. Num grupo de doenças tão extenso e com grande sobreposição e variabilidade fenotípica e genotípica, a pesquisa genética individual e sequencial facilmente se tornam num processo moroso e complexo nas decisões de priorização clínica das várias entidades. ${ }^{7}$ A abordagem sequencial resulta frequentemente num diagnóstico tardio, stress e desânimo por parte do médico e do utente, e numa longa e dispendiosa lista de testes genéticos individuais inconclusivos.

O GNgen do CHSJ recomenda a boa prática clínica do diagnóstico baseado numa entrevista clínica e exame físico exaustivos que permitam orientar uma sequência lógica e eficiente de exames complementares de diagnóstico antes de partir para a realização de estudos complexos e dispendiosos como os painéis genéticos. De facto, mesmo a escolha destes painéis exige um conhecimento clínico exaustivo de cada caso e dos potenciais diagnósticos, devendo ser sempre orientado por especialistas na respetiva área das doenças neurodegenerativas. ${ }^{7}$ A melhor estratégia de priorização do estudo molecular, incluindo os painéis genéticos, deve orientar-se pela identificação de detalhes fenotípicos com valor discriminativo e outros elementos incluindo idade, sexo, etnia e origem geográfica. ${ }^{1,5,7,10,12} \mathrm{O}$ GNgen do CHSJ alerta ainda para o risco da utilização indiscriminada destes painéis e em especial para a problemática das variantes de significado incerto e outros achados inconclusivos, já característicos dos estudos moleculares, mas exponenciados à escala dos painéis genéticos. De facto, é típico encontrar dezenas de variantes (ou mutações) em diversos genes em cada painel realizado, mas normalmen- te só um poderá, na melhor das hipóteses, representar o diagnóstico. A decisão final depende de geneticistas experientes e da ajuda da bioinformática, mas acima de tudo, da capacidade do clínico transmitir a informação fenotípica detalhada e posteriormente confrontar os resultados com esta mesma informação. O GNgen do CHSJ recomenda a boa prática clínica da discussão multidisciplinar dos casos antes e depois da realização deste tipo de estudos genéticos, reunindo profissionais das várias áreas envolvidas, incluindo genética molecular, e com experiência na patologia em estudo. ${ }^{7}$

Tendo as recomendações prévias em mente, o GNgen do CHSJ considera, mediante as particularidades de cada caso clínico e a lista de diagnósticos suspeitos, que o uso de painéis genéticos pode tornar o processo de diagnóstico mais rápido e económico, com potenciais vantagens para a qualidade de vida do utente (p.e. prognóstico, aconseIhamento genético e potenciais terapêuticas). ${ }^{7} \mathrm{O}$ GNgen do CHSJ recomenda sempre a discussão multidisciplinar destes casos, mas considera que os painéis genéticos devem fazer parte do processo de diagnóstico nas ataxias hereditárias, podendo ser considerados em qualquer fase do diagnóstico complementar se a análise multidisciplinar especializada prever o seu benefício clínico e económico em comparação com a abordagem mais tradicional (p.e. testes genéticos individuais sequenciais). ${ }^{7}$

\section{PROTECÇÃO DE PESSOAS E ANIMAIS}

Os autores declaram que os procedimentos seguidos estavam de acordo com os regulamentos estabelecidos pelos responsáveis da Comissão de Investigação Clínica e Ética e de acordo com a Declaração de Helsínquia da Associação Médica Mundial.

\section{CONFIDENCIALIDADE DOS DADOS}

Os autores declaram ter seguido os protocolos do seu centro de trabalho acerca da publicação de dados.

\section{CONFLITOS DE INTERESSE}

Os autores declaram não terem qualquer conflito de interesse relativamente ao presente artigo.

\section{FONTES DE FINANCIAMENTO}

Este trabalho não foi subsidiado por quaisquer fundos provenientes de entidades no domínio público ou privado

\section{REFERÊNCIAS}

1. Ruano L, Melo C, Silva MC, Coutinho P. The global epidemiology of hereditary ataxia and spastic paraplegia: a systematic review of prevalence studies. Neuroepidemiology. 2014;42:174-83.

2. Coutinho P, Ruano L, Loureiro JL, Cruz VT, Barros J, Tuna A, et al. Hereditary ataxia and spastic paraplegia in Portugal: a population-based prevalence study. JAMA Neurol. 2013;70:746-55.

3. van de Warrenburg BP, van Gaalen J, Boesch S, Burgunder JM, Dürr A, Giunti $P$, et al. EFNS/ENS Consensus on the diagnosis and management of chronic ataxias in adulthood. Eur J Neurol. 2014;21:552-62.

4. Gasser T, Finsterer J, Baets J, Van Broeckhoven C, Di Donato S, Fontaine $\mathrm{B}$, et al. EFNS guidelines on the molecular diagnosis of ataxias

and spastic paraplegias. Eur J Neurol. 2010;17:179-88.

5. Rossi M, Perez-Lloret S, Doldan L, Cerquetti D, Balej J, Millar Vernetti $P$, et al. Autosomal dominant cerebellar ataxias: a systematic review of clinical features. Eur J Neurol. 2014;21:607-15

6. Shakkottaib VG, Fogel BL. Clinical neurogenetics: autosomal dominant spinocerebellar ataxia. Neurol Clin. 2013;31:987-1007.

7. Jayadev S, Bird TD. Hereditary ataxias: overview. Genet Med. 2013;15:673-83.

8. Choi KD, Choi JH. Episodic ataxias: clinical and genetic features. J Mov Disord. 2016;9:129-35.

9. Kim JS, Cho JW. Hereditary cerebellar ataxias: a Korean perspective. J 
Mov Disord. 2015;8:67-75.

10. Beaudin M, Klein CJ, Rouleau GA, Dupre N. Systematic review of autosomal recessive ataxias and proposal for a classification. Cerebellum Ataxias. 2017;4:3.

11. Bras J, Alonso I, Barbot C, Costa MM, Darwent L, Orme T, et al. Mutations in PNKP cause recessive ataxia with oculomotor apraxia type 4. Am J Hum Genet. 2015;96:474-9.
12. Musselman KE, Stoyanov CT, Marasigan R, Jenkins ME, Konczak J, Morton SM, et al. Prevalence of ataxia in children: a systematic review. Neurology. 2014;82:80-9.

13. Wolf NI, Koenig M. Progressive cerebellar atrophy: hereditary ataxias and disorders with spinocerebellar degeneration. Handb Clin Neurol. 2013;113:1869-78. 